\title{
Electro-spraying and ultra-violet light curing of polydimethylsiloxane to fabricate thin films for low-voltage dielectric elastomer actuators
}

\author{
Florian M. Weiss ${ }^{\mathrm{a}, \mathrm{b}}$, Gabor Kovacs ${ }^{\mathrm{a}}$, Tino Töpper ${ }^{\mathrm{b}}$, Bekim Osmani ${ }^{\mathrm{b}}$, Vanessa Y. F. Leung ${ }^{\mathrm{b}}$, and Bert \\ Müller*b \\ ${ }^{a}$ Swiss Federal Laboratories for Materials Science and Technology, Überlandstrasse 129, 8600 \\ Dübendorf, Switzerland; ${ }^{b}$ Biomaterials Science Center, Department of Biomedical Engineering, \\ Medical Faculty, University of Basel, 4123 Allschwil, Switzerland.
}

\begin{abstract}
Currently, dielectric elastomer actuators (DEA) are mainly based on micrometer-thin polymer films and require operating voltages of several hundred volts. In medical applications, however, voltages as low as a few tens of volts are required. To this end, we prepared nanometer-thin dielectric elastomer layers. It is demonstrated that alternating current, electro-spray deposition allows for the fabrication of homogenous, flat, nanometer-thin polydimethylsiloxane (PDMS) films. The growth of the PDMS with average number molecular weights ranging from 800 to $62,700 \mathrm{~g} / \mathrm{mol}$, at a constant flow rate of $267 \mathrm{~nL} / \mathrm{s}$, was in situ monitored by means of spectroscopic ellipsometry. The Cauchy layer model used for data interpretation may only be applied to flat PDMS layers. Thus, in the present study the droplet morphology was also determined by atomic force microscopy. Spectroscopic ellipsometry does allow for the qualitative determination of the thin film morphology. However, for high molecular weight polymers the precise measurement during deposition is challenging. Independent of the molecular weight, the roughness of the deposited PDMS films considerably smoothens during the ultra-violet radiation treatment. After curing, the electro-sprayed nanometer-thin PDMS films are homogeneous enough to qualify for the fabrication of low-voltage DEA.
\end{abstract}

Keywords: Ellipsometry, nanometer-thin film, atomic force microscopy, surface roughness, artificial muscle, incontinence.

\section{INTRODUCTION}

Electrospray ionization (ESI) well known from mass spectroscopy (MS) as a soft ionization method for large (bio-) molecules [1] found its application also as deposition method. Electrospray deposition (ESD) has attracted attention for decades. There are numerous investigations on thin, dense or porous film deposition [2] for a variety of applications as well as nanometer-sized particle formation or encapsulations [3]. Not only inorganic and organic materials were deposited for direct applications but also a lot of effort was taken to understand the behavior of the spray mechanism with respect to the influencing parameters from the setup and the sprayed solution [4-7]. Furthermore, studies on the deposit morphology dependence on flow rate and vapor pressure have been carried out for polymers $[8,9]$.

Usually the investigation on the electrospray, whether for ionization or deposition, is described in its original direct current (dc), static electric field, mode. Considering the deposition, this means that the substrate has to be conductive, so that the nozzle represents one electrode and the substrate the counter electrode. For deposition on non-conductive substrates alternating current (ac) electrospray deposition has been suggested and successfully applied [10]. Up to now a restricted number of studies has been published. This is due to the spray stability and the frequent application of conductive substrates.

Investigations on dielectric elastomer transducers (DET) aim to enhance the materials and designs according to their applications. For dielectric elastomer actuators (DEAs), in particular, lowering the operation voltages from the currently used values of several hundred volts to physiologically relevant ones is essential for medical implants [11]. Here, one may either increase the permittivity of the dielectric material sandwiched between the electrodes or reduce its layer thickness below one micrometer [12]. Innumerable investigations were conducted on the materials permittivity [11]. Conversely, only a few studies on film thickness reduction are known [13-15]. To compensate for the low forces, which

*Bert Müller, bert.mueller@unibas.ch,+41 61265 9660, www.bmc.unibas.ch

Electroactive Polymer Actuators and Devices (EAPAD) 2016, edited by Yoseph Bar-Cohen, Proc. of SPIE Vol. 9798, 97983C · C 2016 SPIE · CCC code: 0277-786X/16/\$18 · doi: 10.1117/12.2220786 
Here, we propose the ac electrospray method as an approach for future fabrication of nanometer-thin elastomer layers in low-voltage DEAs with the aim to realize artificial muscles for incontinence treatment $[16,17]$. For this application, the films have to be quantitatively characterized, which includes the surface roughness of the PDMS films as the function of the deposition rate.

\section{WORKING PRINCIPLES ELECTROSPRAY DEPOSITION}

\subsection{DC electrospray deposition}

In the direct current $(\mathrm{dc})$ mode a static electric field between the nozzle and the conducting substrate is applied using a high voltage (HV) source. The molecules in the solution become charged. The charging causes a separation along the electric field. If for example, a positive charge is applied on the nozzle, the positively charged molecules will be attracted towards the negatively charged substrate. This, in addition to the solution pressure, usually induced by gravity or a syringe pump, forces the solution to flow out of the nozzle to the substrate. Once the applied electric field exerts a force similar to the surface tension, the normally observed drop formation vanishes and a cone, termed TAYLOR cone, is formed. At this stage, with the solution pressure a cone jet is formed, which eventually breaks up into charged droplets. During descending the droplets diminish their volume caused by solvent evaporation, whereas the charge stays constant. This increase in charge to volume ratio will eventually reach a critical value, termed RAYLEIGH limit. It is the point the surface tension equals the repulsive forces of the charged molecules. If the critical charge to volume surface is exceeded, the droplets undergo the CoUlOMB explosion, i.e. a fission into smaller droplets. This process is repeated for several times until the droplets impinge on the surface. On the conductive substrate, they will become neutralized, see Figure 1(a). This process depends on many parameters of the applied solution namely the surface tension, the vapor pressure, the electric conductivity and the viscosity. In addition, the setup plays a critical role, as it determines the strength of the applied electric field, which relates to the nozzle-substrate distance.
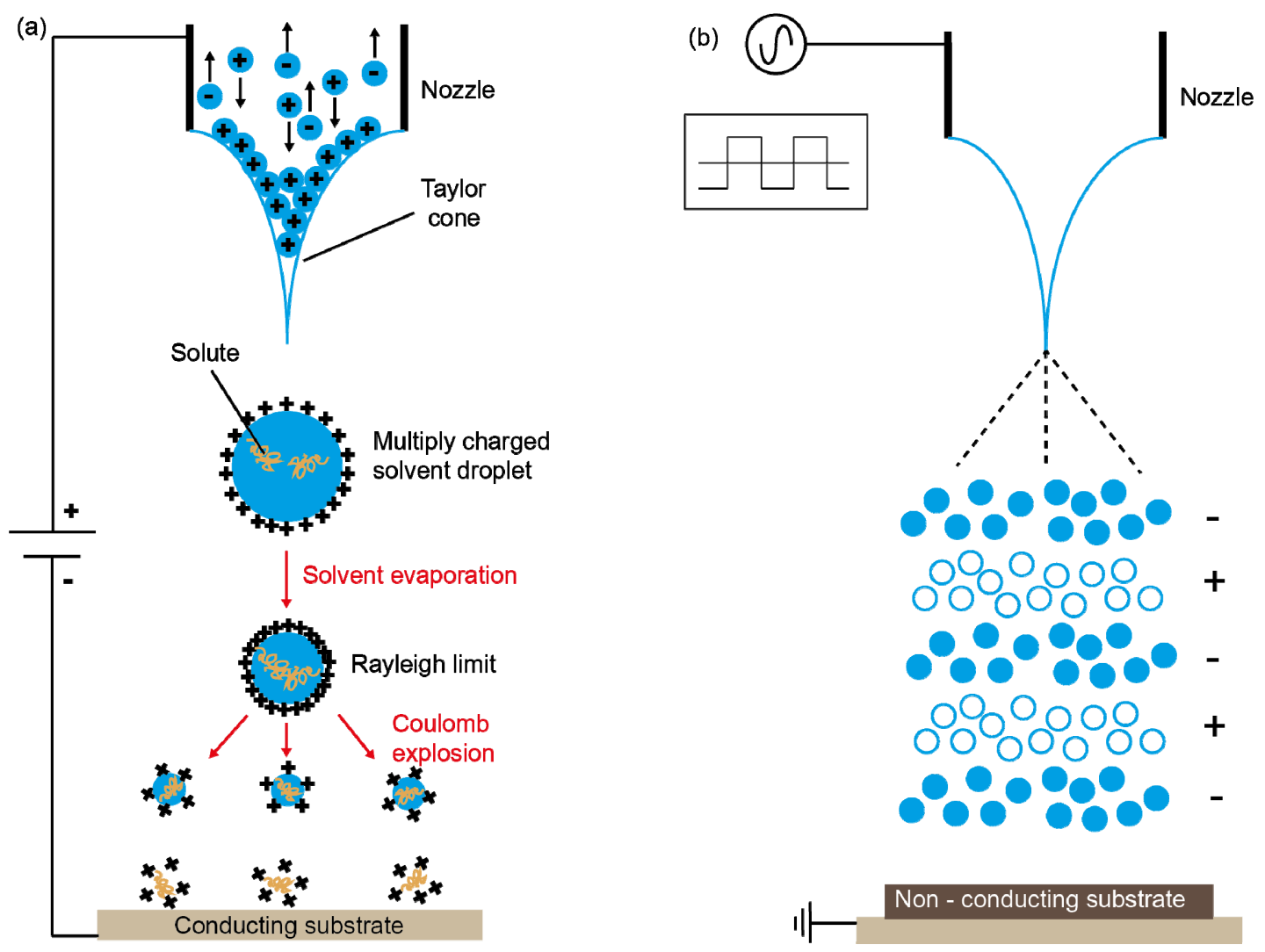

Figure 1. The schemes represent the basic principles of the electrospray deposition in the dc (a) and ac (b) modes. 


\subsection{AC electrospray deposition}

The alternating current (ac) mode for deposition is usually only applied, if the substrate is electrically insulating [10]. In this mode, an alternating electric field is applied. It avoids surface charge accumulation on the substrate. Clouds of charged droplets are emitted, as illustrated in Figure 1(b), which finally neutralize each other on the substrate. Therefore, relatively thick layers can be deposited without repulsion interactions between droplets and substrate. Owing to the alternating field it is more difficult to obtain stable conditions. In this mode, an additional parameter for the deposition control, i.e. the frequency of polarity alternation, has to be considered. The limited number of available studies on the ac mode show the influence of frequency and reveal the applicability of this alternative approach [18-20].

\section{EXPERIMENTAL}

\subsection{Material and electro-spray deposition}

The spray solution contained 5 vol.\% telechelic vinyl PDMS polymers (Gelest, USA) with molecular weights $M_{n}$ of 800 , 6,000, 28,000, and 62,700 g/mol (DMS-V05, DMS-V21, DMS-V31, and DMS-V41, respectively) dissolved in ethyl acetate (Laboratory reagent grade, Fisher Scientific UK, Brunschwig, Basel, Switzerland). Solvent and PDMS polymers were not further purified. Using a $3 \mathrm{~mL}$ glass syringe with a metallic Leur-lock (Eternal-Matic, Sanitex, HUBERLAB, Aesch, Switzerland) connected to a metallic nozzle (26s, I.D. $0.13 \mathrm{~mm}$, Hamilton, Bonaduz, Switzerland) the solution was extruded by a syringe pump (Aladdin six-syringe pump, World Precision Instruments Germany GmbH, Berlin, Germany) at a constant flow rate of $267 \mathrm{~nL} / \mathrm{s}$ for the present experiments. Applying a constant alternating voltage of $\pm 5 \mathrm{kV}$ with a frequency of $18 \mathrm{~Hz}$ using a voltage source (TREK, 5/80, Lockport, NY, USA) and a function generator (Model 119M, Max Meier Elektronik, Zurich, Switzerland) a rectangular voltage function was obtained, monitored by a Tektronix oscilloscope (TDS 210, Computer Controll AG, Zurich, Switzerland). The chosen distance from the the nozzle to the Si substrate (SiMat, Silicon Materials, Kaufering, Germany) amounts $67 \mathrm{~mm}$. The UV radiation from a deuterium broad-band UV lamp (Yuyu Lightning, China) with a spectral range between 180 and $450 \mathrm{~nm}$, and a maximum intensity at $210 \mathrm{~nm}$ at a distance of $2 \mathrm{~cm}$ induced the curing without any photo-initiator. Curing was performed in an Ar (Carbagas AG, Basel, Switzerland) atmosphere of 1 bar, see Figure 2.

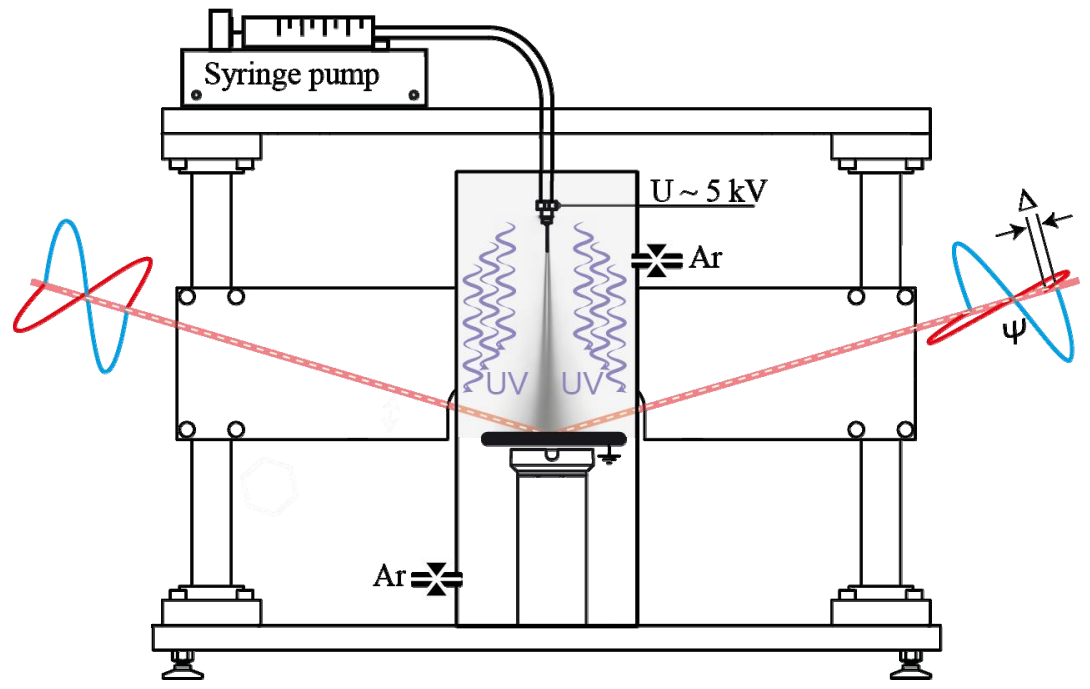

Figure 2. The experimental setup of the in-house built ac electrospray deposition system coupled with spectroscopic ellipsometry for real-time data acquisition in situ.

\subsection{Real-time spectroscopic ellipsometry}

To monitor the film growth and the curing process in real time, the spectroscopic ellipsometer SE801 from the company SENTECH (Berlin, Germany), controlled by the SpectraRay3 software, was used. The relevant angles $\Psi$ and $\Delta$ were measured in the spectral range between 300 to $1050 \mathrm{~nm}$. The incident beam angle was held constant at $70^{\circ}$ from the normal, cp. Figure 2. The $2 \mathrm{~mm}$ beam diameter resulted in a spot size of $2 \mathrm{~mm} \times 5 \mathrm{~mm}$ on the substrate. The real time 
measurement period was set to $1 \mathrm{~s}$ steps. The linear and first order non-linear refractive indices as determined by a static measurement of a spin-coated, $2 \mu \mathrm{m}$-thick film of the DMS-V21, revealed values of $n_{0}=1.396 \pm 0.005$ and $n_{1}=37 \pm 0.7$, respectively, and were set as constant values for all measurements. The wavelength-independent extinction coefficient $k_{0}$ was used to simulate the scattering at the surface and, therefore, as a phenomenological parameter for the surface roughness.

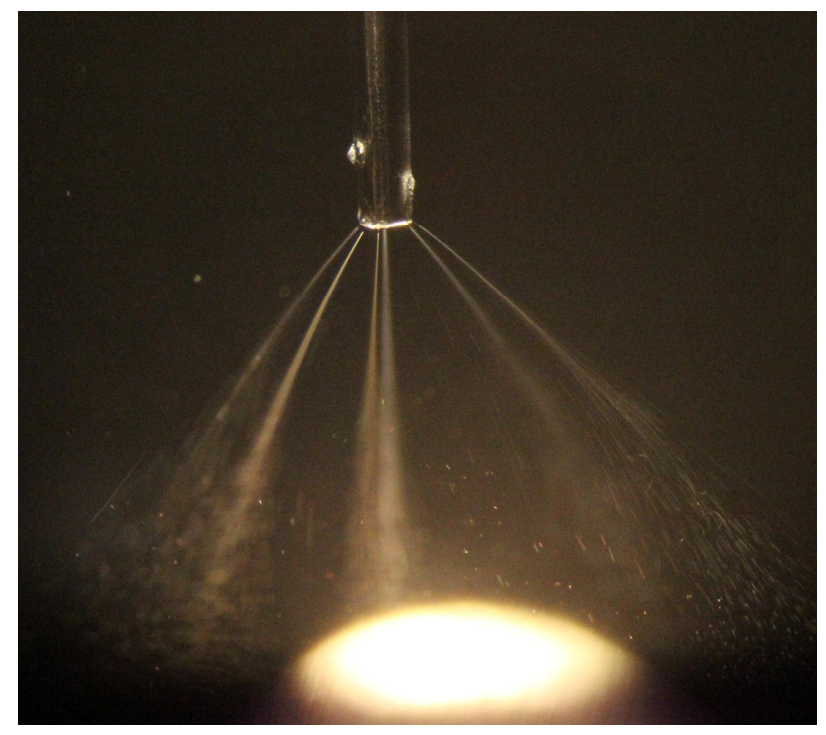

Figure 3. Exemplary optical micrograph of a multi-cone jet spray as experimentally observed.

\subsection{Atomic force microscopy}

AFM scans of $90 \times 90 \mu \mathrm{m}^{2}$ areas were acquired in tapping mode with a vibration amplitude of $1774 \mathrm{mV}$ and a set point of $20 \%$ on a FlexAFM system (Nanosurf AG, Liestal, Switzerland). We have used a Tap 190Al-G AFM probe (BudgetSensors, NanoAndMore GmbH, Wetzlar, Germany) to acquire 512 lines at a speed of $2 \mathrm{~s}$ per line. The raw data were processed using the Gwyddion 2.41 software package (Gwyddion: an open-source software for SPM data analysis, http://gwyddion.net).

\section{RESULTS AND DISCUSSION}

\subsection{Deposition process}

The evaluation of the recorded data by spectroscopic ellipsometry was only accounted in the spectral range of 300 to $1050 \mathrm{~nm}$, because the absorption of PDMS is pronounced for wavelengths below $300 \mathrm{~nm}$ [21]. The baseline was taken from an ellipsometric measurement of a bare $\operatorname{Si}(100)$ substrate having a 2 to $3 \mathrm{~nm}$-thin native oxide layer. Both, the native oxide and the sprayed PDMS, were considered as Cauchy layers in order to extract the optical properties and the thickness of the growing film. The monitored $\Delta$ - and $\Psi$-data are related to the complex Fresnel reflection coefficients $r_{\mathrm{p}}$ and $r_{\mathrm{s}}$ of $\mathrm{p}$ - and s-polarized light and their ratio $\rho$, described by the Fresnel equation

$$
\rho=r_{p} / r_{s}=\tan \Psi \cdot e^{i \Delta}
$$

To obtain the dielectric function $\tilde{n}(\lambda)$ from the measured data equation (2) can be used:

$$
\langle\tilde{n}\rangle^{2}=(\langle n\rangle+i\langle k\rangle)^{2}=\sin \left(\varphi_{0}\right)^{2} \cdot\left(1+\tan \left(\varphi_{0}\right)^{2}\left(\frac{1-\rho}{1+\rho}\right)^{2}\right)
$$


where $n(\lambda)$ is the real and $k(\lambda)$ the complex part of the refractive index. $\varphi_{0}$ represents the angle of the incident beam. The simplest approximation according to the Cauchy series is to use the wavelength-independent, constant extinction coefficient

$$
k(\lambda)=k_{0}
$$

and to reduce the refractive index to

$$
n(\lambda)=n_{0}+c_{1} n_{1} / \lambda^{2}
$$

with $c_{1}=100 \mathrm{~nm}^{2}$. Quantitatively the overlap of the the experimental $\Delta$ - and $\Psi$-data and the model has been evaluated by the mean square error (MSE)

$$
\frac{1}{N} \sqrt{\sum_{i=1}^{N}\left\{\left(\frac{\Psi_{i}^{m}-\Psi_{i}^{t h}}{\delta \Psi}\right)^{2}+\left(\frac{\Delta_{i}^{m}-\Delta_{i}^{t h}}{\delta \Psi}\right)^{2}\right\}}
$$

During the initial stages of growth, however, the fitting according to the Cauchy layer model nor the fitted data by the effective medium layer model [22] were reasonable, presumably because of the droplets present on the substrate. For the higher molecular weight polymers DMS-V31 and DMS-V41 no acceptable fits could be obtained during the whole spraying process. For the polymers DMS-V05 and DMS-V21 at advanced stages of growth, the fitting according to the Cauchy layer model becomes reasonable. Hence, we assume that the droplets start to form a confluent layer with a rough surface morphology represented by the fitted constant extinction coefficient $k_{0}$. Here, $k_{0}$ simulates the scattering and the absorption of the incident beam at the protrusions on the growing surface. Thus, $k_{0}$ rather describes a phenomenological parameter due to the damping of the $\Delta$ - and $\Psi$-amplitudes. Therefore, we neglected the wavelength dependency of $k$.

Figure 4 presents the extinction coefficient monitored using ellipsometry versus the deposition time or the equivalent layer thickness obtained for DMS-V05 and DMS-V21. For the polymer DMS-V05, the onset of the fit derived from the Cauchy model starts well below a film thickness of $100 \mathrm{~nm}$. For the polymer DMS-V21 the onset is observed above $500 \mathrm{~nm}$. It agrees with measurements published recently [15]. Furthermore, it is obvious that the measurements show a surface roughness decrease, as indicated by the decreasing value of $k_{0}$ as the thickness increases.

Time $t(\mathbf{s})$

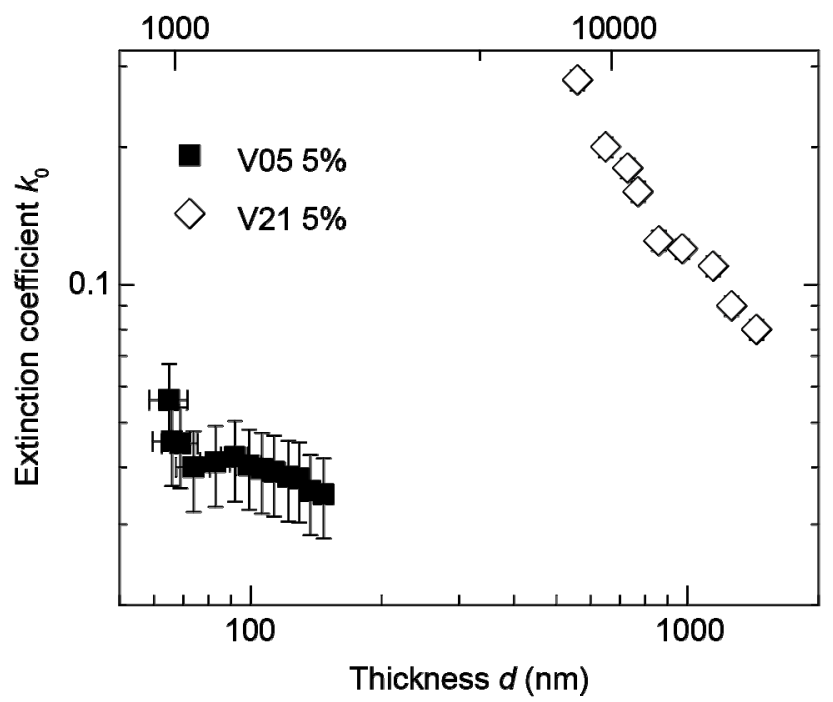

Figure 4. The extinction coefficient $k_{0}$ plotted against the layer thickness $d$ and deposition time $t$ obtained by real-time ellipsometry for $5 \%$ DMS-V05 and DMS-V21 in solution.

The reason for the incommensurability of the high molecular weight polymers DMS-V31 and DMS-V41 is attributed to their droplet size and shape as shown in the AFM images of Figure 5. Due to the height of hundreds of nanometers or 
even in the micrometer range we assume an immense light scattering causing the damping of interferences within the $\Delta$ and $\Psi$ - data. This behavior hinders a reasonable thickness extraction from the data analysis. Additionally, we point out that droplets with hundreds of nanometer in height are not favorable for nanometer-thin film production. Note that the droplets shown in Figure 5 are already cured. Nevertheless, they are expected to show similarities to the island shapes formed by the impinged droplets before curing. For DMS-V05 and DMS-V21 the droplet-like islands are low, a few tens of nanometers in height, and they show plateau-like surfaces, which also supports the signal collection for ellipsometry, see Figure 4.

The early detection onset as well as the low surface roughness of DMS-V05 are attributed to the high mobility and superior wettability of the short polymer chains compared to the significantly longer chains of DMS-V21, see Table 1. It should be considered though that all the polymer samples are used as purchased and, therefore, contain a polymer size distribution, which has an influence on the mobility and wetting behavior.

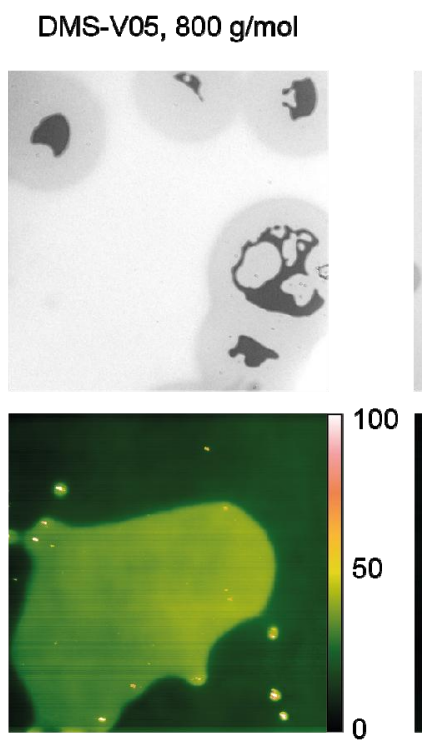

DMS-V21, 6'000 $\mathrm{g} / \mathrm{mol}$
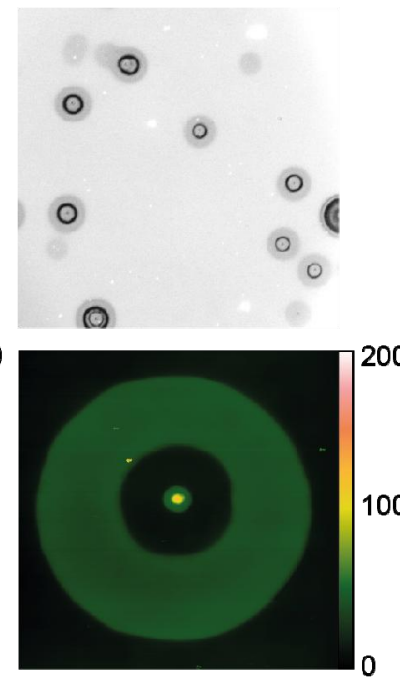

DMS-V31, 28 '000 g/mol

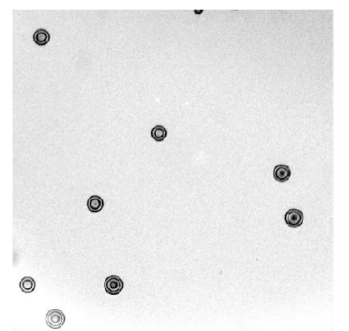

200

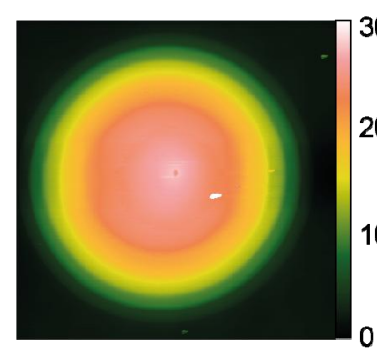

DMS-V41, $62 ' 700 \mathrm{~g} / \mathrm{mol}$

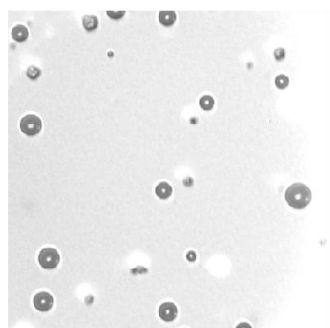

300

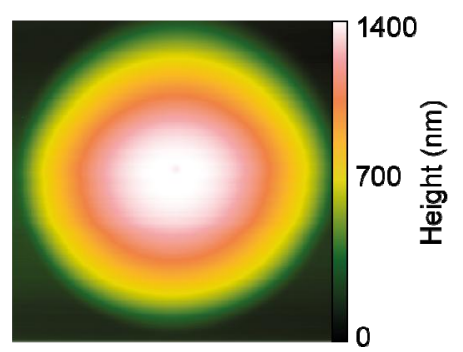

Figure 5. Optical micrographs $\left(2.34 \times 2.34 \mathrm{~mm}^{2}\right)$ and related AFM images $\left(90 \times 90 \mu \mathrm{m}^{2}\right)$ of sprayed droplets from the four polymer solutions prepared, as indicated, after UV light curing.

Comparing the morphology of islands formed after UV curing, one recognizes distinct features, see Figure 5. The origin of the characteristic features has been discussed previously [14], and are referred to the chain length or the related viscosity. Comparing the morphology of the DMS-V05 and DMS-V21 polymer islands, the possibility of ellipsometry analysis for the lower molecular weight polymer at relatively low coverage is evident.

\subsection{Ultra-violet light curing of the electro-sprayed films}

Subsequent to deposition, the films could relax for about one hour. During this period of time, the remaining solvent can evaporate, and the droplet-like islands spread out to reach an equilibrium state. In a next step, the films were treated with UV radiation in argon atmosphere. During the curing ellipsometry data were collected for the four selected polymers. Some results are shown in Figure 6. The extinction coefficient is plotted against the deposition time. The red-colored fit fitted lines represent the exponential decay. Time constants $\tau$ can be extracted from these fits and are summarized in column 3 of Table 1.

UV light treatments induce film smoothening quantified by the decrease of the extinction coefficient. The final values range from 0.012 to 0.045 , which is even below of the earlier published values $(0.04$ to 0.08$)$ [15]. One has to consider that the areas here were smaller compared to the previous study because of the geometry of the experimental setup. The time constants derived show an increase with molecular weight. The experimentally observed behavior is expected, as the smaller molecules can migrate faster than the larger ones. 


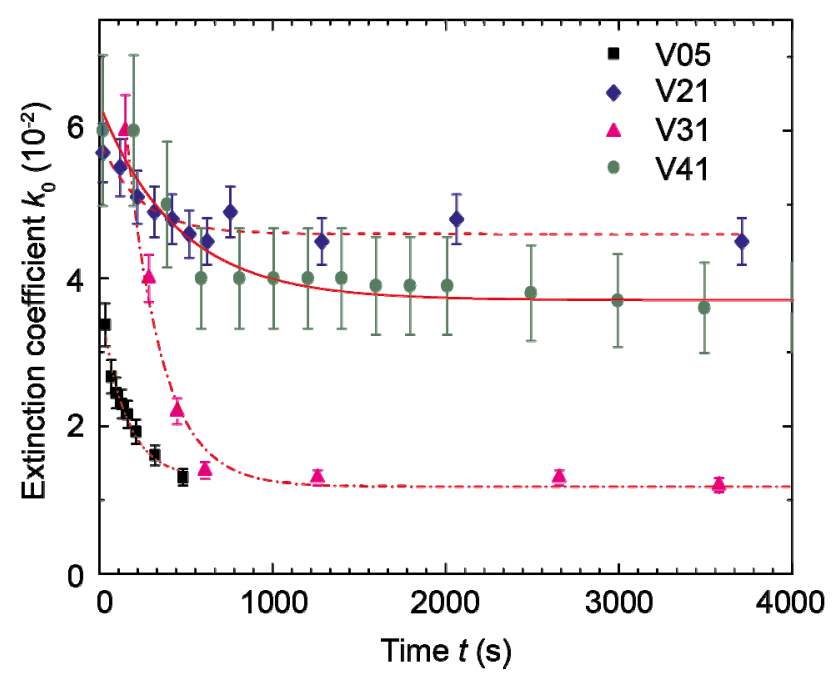

Figure 6. Real-time spectroscopic ellipsometry measurements during UV light curing in Ar atmosphere are shown. The diagram presents the extinction coefficient $k_{0}$ versus the curing time $t$ for the selected polymer materials with the fits given by red-colored lines. The fits of exponential decay provide the time constant $\tau$ of the curing process.

Table 1. Summary of viscosity of the processed polymers, film thickness and detected time constant $\tau$ of the UV curing process extinction coefficient $k_{0}$ are presented.

\begin{tabular}{|c|c|c|c|}
\hline Polymer & Viscosity (cSt.) & Layer thickness (nm) & $\boldsymbol{\tau}\left(\boldsymbol{k}_{\mathbf{0}}\right)$ \\
\hline DMS-V05 & $4-8$ & $155 \pm 4$ & $96 \pm 8$ \\
\hline DMS-V21 & 100 & $1,440 \pm 43$ & $158 \pm 11$ \\
\hline DMS-V31 & 1,000 & $455 \pm 23$ & $394 \pm 32$ \\
\hline DMS-V41 & 10,000 & $905 \pm 27$ & $339 \pm 58$ \\
\hline
\end{tabular}

In summary, the electrospray deposition and subsequent UV light curing results in flat nanometer-thin films for the selected polymer starting materials. The chain length and the molecular weight, respectively, influence the success of real-time spectroscopic ellipsometry during film formation.

It should be noted that the technique is promising but needs further elaboration to optimize the process parameters. The experiments were performed with fixed electric field and flow rate. As shown in Figure 3, the spray showed instable cone jets. Thus the films are rather inhomogeneous considering the area of an entire 2-inch wafer. Multiple nozzle arrangements and moving and/or rotating substrates may further improve the homogeneity.

\section{CONCLUSIONS}

AC electrospray deposition is a favorable technique for future nanometer-thin film production of polymers for lowvoltage dielectric elastomer actuators and sensors. UV light curing reduces the roughness of the PDMS films below one nanometer. The processes can in situ be monitored using advanced ellipsometry and related data evaluation. Large-area deposition with improved homogeneity should be reached through multi-nozzle arrays and substrate movements. Therefore, AC electrospray deposition is the only known method, which shows potential for industrial applications in the field of low-voltage DEA fabrication. 


\section{ACKNOWLEDGEMENT}

The authors gratefully acknowledge the financial support of the Swiss National Science Foundation (project 200021135496) and the nano-tera.ch initiative (project SmartSphincter) as well as the Swiss Nanoscience Institute (SNI) for the financial contribution to the AFM.

\section{REFERENCES}

[1] Fenn, J. B., Mann, M., Meng, C. K. et al., "Electrospray ionization for mass spectrometry of large biomolecules," Science, 246(4926), 64-71 (1989).

[2] Jaworek, A., "Electrospray droplet sources for thin film deposition,” J. Mater. Sci., 42(1), 266-297 (2007).

[3] Jaworek, A., and Sobczyk, A. T., "Electrospraying route to nanotechnology: An overview," J. Electrostat., 66(3), 197-219 (2008).

[4] Cloupeau, M., and Prunet-Foch, B., "Electrostatic spraying of liquids: Main functioning modes," J. Electrost., 25(2), $165(1990)$.

[5] Ganan-Calvo, A. M., "On the general scaling theory for electrospraying," J. Fluid Mech., 507, 203 (2004).

[6] Ganan-Calvo, A. M., Davila, J., and Barrero, A., "Current and droplet size in the electrospraying of liquids. Scaling laws," J. Aerosol Sci., 28(2), 249 (1997).

[7] Hartman, R. P. A., Brunner, D. J., Camelot, D. M. A. et al., "Electrohydrodynamic atomization in the cone-jet mode physical modeling of the liquid cone and jet," J. Aerosol Sci., 30(7), 823 (1999).

[8] Rietveld, I. B., Kobayashi, K., Yamada, H. et al., "Morphology control of poly(vinylidene fluoride) thin film made with electrospray,” J. Colloid Interface Sci., 298(2), 639 (2006).

[9] Rietveld, I. B., Kobayashi, K., Yamada, H. et al., "Model supported morphology control of electrospray deposited poly(vinylidene fluoride) film," Macromolecular Symposia, 249, 322 (2007).

[10] Sarkar, S., Levit, N., and Tepper, G., "Deposition of polymer coatings onto saw resonators using ac electrospray," Sens. Actuators B Chem., 114(2), 756-761 (2006).

[11] Madsen, F. B., Daugaard, A. E., Hvilsted, S. et al., "The current state of silicone-based dielectric elastomer transducers," Macromol. Rapid Commun., 37(5), 378-413 (2016).

[12] Heydt, R., Kornbluh, R., Eckerle, J. et al., [Dielectric elastomers as electromechanical transducers: Fundamentals, materials, devices, models and applications of an emerging electroactive polymer technology] Elsevier, Oxford (2008).

[13] Töpper, T., Weiss, F., Osmani, B. et al., "Siloxane-based thin films for biomimetic low-voltage dielectric actuators," Sens. Actuators A Phys., 233, 32-41 (2015).

[14] Weiss, F. M., Töpper, T., Osmani, B. et al., "Thin film formation and morphology of electro-sprayed polydimethylsiloxane," Langmuir, (2016).

[15] Weiss, F. M., Töpper, T., Osmani, B. et al., "Electrospraying nanometer-thin elastomer films for low-voltage dielectric actuators," Adv. Electron. Mater., 1-8 (2016).

[16] Fattorini, E., Brusa, T., Gingert, C. et al., "Artificial muscle devices: Innovations and prospects for fecal incontinence treatment," Ann. Biomed. Eng., 1-15 (2016).

[17] Müller, B., Deyhle, H., Mushkolaj, S. et al., "The challenges in artificial muscle research to treat incontinence," Swiss Med. Wkly., 139(41-42), 591-595 (2009).

[18] Yeo, L. Y., Lastochkin, D., and Chang, H.-C., "High frequency ac electrospraying of dielectric liquids," ASME 2004 2nd International Conference on Microchannels and Minichannels, 723-730 (2004).

[19] Yeo, L. Y., Lastochkin, D., Wang, S.-C. et al., "A new ac electrospray mechanism by maxwell-wagner polarization and capillary resonance," Phys. Rev. Lett., 92(13), 133902 (2004).

[20] Maheshwari, S., and Chang, H.-C., "Anomalous conical menisci under an ac field-departure from the dc taylor cone,” Appl. Phys. Lett., 89(23), 234103 (2006). 
[21] Lee, J., Kim, J., Kim, H. et al., "Effect of thermal treatment on the chemical resistance of polydimethylsiloxane for microfluidic devices," J. Micromech. Microeng., 23(3), 035007 (2013).

[22] Bruggeman, D. A. G., "Berechnung verschiedener physikalischer konstanten von heterogenen substanzen. I. Dielektrizitätskonstanten und leitfähigkeiten der mischkörper aus isotropen substanzen,” Ann. Phys., 416(8), 665 (1935). 